\title{
Joint Coding and Scheduling Optimization in Wireless Systems with Varying Delay Sensitivities
}

\author{
Weifei Zeng \\ Research Laboratory of Electronics, \\ Massachusetts Institute of Technology, \\ Cambridge, MA, USA, \\ Email: weifei@mit.edu
}

\author{
Chris T. K. Ng \\ Bell Laboratories, \\ Alcatel-Lucent, \\ Holmdel, NJ, USA, \\ Email: chris.ng@alcatel-lucent.com
}

\author{
Muriel Médard \\ Research Laboratory of Electronics, \\ Massachusetts Institute of Technology, \\ Cambridge, MA, USA, \\ Email: medard@mit.edu
}

\begin{abstract}
Throughput and per-packet delay can present strong trade-offs that are important in the cases of delay sensitive applications. We investigate such trade-offs using a random linear network coding scheme for one or more receivers in single hop wireless packet erasure broadcast channels. We capture the delay sensitivities across different types of network applications using a class of delay metrics based on the norms of packet arrival times. With these delay metrics, we establish a unified framework to characterize the rate and delay requirements of applications and optimize system parameters. In the single receiver case, we demonstrate the trade-off between average packet delay, which we view as the inverse of throughput, and maximum ordered inter-arrival delay for various system parameters. For a single broadcast channel with multiple receivers having different delay constraints and feedback delays, we jointly optimize the coding parameters and time-division scheduling parameters at the transmitters. We formulate the optimization problem as a Generalized Geometric Program (GGP). This approach allows the transmitters to adjust adaptively the coding and scheduling parameters for efficient allocation of network resources under varying delay constraints. In the case where the receivers are served by multiple non-interfering wireless broadcast channels, the same optimization problem is formulated as a Signomial Program, which is NP-hard in general. We provide approximation methods using successive formulation of geometric programs and show the convergence of approximations.
\end{abstract}

Index Terms-Network Coding, Delay, Throughput, Optimization, Geometric Programming

\section{INTRODUCTION}

The growing diversity of network applications, protocols and architectures poses new problems related to the fundamental trade-offs between throughput and delay in communications. For instance, applications like file downloading or FTP protocols aim solely to maximize transmission rate and to minimize the overall completion time. On the other hand, applications such as real-time video conferencing are highly sensitive to delay of any consecutive packets. Failure to meet continuous delivery deadlines in stream of packets quickly deteriorates the Quality of user Experience (QoE). The two extremes in delay sensitivities by no means represent all types of applications. Progressive downloading video, for example, would be more delay sensitive than file downloading, but less sensitive than real-time video streaming, since the receiver has buffered sufficient content.

In this paper, we develop a unified framework to study rate and delay trade-offs of coding and scheduling schemes and to optimize their performance for applications with different delay sensitivities. We use a class of delay metrics based on the $\ell_{p}$-norms of the packet arrivals times to represent delayrate characteristics and requirements of applications. At one extreme, the delay metric could capture the average delay and thus the rate of transmission. At the other extreme, the metric measures the maximum ordered inter-arrival delay. Based on the delay metrics, we look to optimize coding and scheduling parameters in a networking system, where various devices with different delay requirements are served by single-hop wireless erasure broadcast channels, each associated with an access point (AP).

The coding scheme in this paper is a variation of the generation-based random linear network coding, presented in [1] and [2]. Specifically, the sender maintains a coding bucket for each receivers. When a transmitter is ready to send a packet to some receiver, it reads the all the packet in the coding bucket for the receiver and produces an encoded packet by forming a random linear combination of all the packets in the coding bucket. The encoded packet is then broadcasted to all the receivers. Once a receiver collects enough packets to decode all packets in the coding bucket through Gaussian elimination, it uses a separate feedback channel to send an ACK message back to the sender. The sender always receives the ACK message after a certain delay. It then purges all the packets in the coding bucket and moves new packets into the bucket. The respective delay constraints of the receivers are known to the sender, who determines adaptively the number of packets to put in the coding buckets for each receiver, by solving system-wise optimization problems. A precise description of the transmission scheme is given in Section П. The coding buckets act as the Head of Line (HOL) generations in the most generation based scheme. However, unlike most generation-based schemes, packets are not partitioned prior to transmission and the bucket sizes in our scheme may vary over time and across different receivers, depending on each receiver's changing delay constraints. The coding parameters are optimized jointly with time division resource allocation parameters to exploit the trade-offs between rate and delay. We first illustrate the trade-offs in the case of point-to-point erasure channels. Then, in the case of multiple receivers 
with one AP, we formulate the delay constrained optimization problem as a Generalized Geometric Program, which can be very efficiently solved. We compare the solutions with fixed generation size schemes for specific examples. Finally, in the case of multiple APs with non-interfering erasure broadcast channels, we formulate the problem as a Signomial Program and provide methods to approximate this non-convex optimization with successive GPs.

There exists a significant amount of related literature and we shall only examine a incomplete set of relevant ones.Previous work by Walsh et al. [3] considers the rate and delay tradeoff in multipath network coded networks, while [4] studies the related issue of rate-reliability and delay trade-off by constructing various network utility maximization (NUM) problems. The concept of network coding is introduced in [5] and linear network coding is extensively studied in [6] and [7]. Other typical rateless codes that are asymptotically optimal for erasure channels are seen in [8] [9] [10]. However, unlike linear network codes which allow intermediate nodes to recode packets, the class of fountain codes are generally only used for one-hop communication systems, as the packets can not be recoded due to stringent packet degree distribution requirements. In our system, the delay constraints make it difficult to apply fountain codes efficiently, as the asymptotic optimality is only achieved with coding over relatively large number of packets. On the other hand, we have feedback which will allow us to dynamically change the coding parameters. The network coding gain in overall delay of file downloading with multicast over packet erasure broadcast channel is characterized in [11] and [12]. With the use of similar linear network codes, broadcast coding schemes based on perfect immediate feedback are proposed and their delay characteristics are analyzed in [13] [14] [15]. An analysis of random linear codes with finite block size is given in [16].

The remainder of the paper is organized as follows. Section [II introduces our model, the code and delay metrics, as well as how the metrics apply specifically to the coding scheme. Section III gives a concise primer on Geometric Programming (GP), which is the basic tool for solving our optimization program. Section IV considers a single wireless broadcast channel with packet erasures. We construct a joint optimization program, which is solved using GP techniques. Furthermore we illustrate the delay and throughput trade-offs with different system parameters and compare the solutions with fixed generation size schemes. Section $\nabla$ extends on the results to multiple non-interfering wireless channels. We provide approximation algorithms to the non-convex optimization problem in this case. Section VI concludes the paper.

\section{Delay Metrics AND Coding}

\section{A. Adaptive Linear Coding Scheme}

Consider a point-to-point communication system illustrated in Figure 1(a) The sender (Tx) and the receiver (Rx) are connected by a wireless erasure channel with packet erasure probability $\varepsilon$ and a perfect feedback channel with delay $D$. The sender looks to transmit to the receiver a flow $f$ consisting of
$N$ packets. The packets are denoted as $\left\{P_{1}^{f}, \cdots, P_{N}^{f}\right\}$. Each of them is treated as a length $m$ vector in the space $\mathbb{F}_{q}^{m}$, over some finite field $\mathbb{F}_{q}$. All $N$ data packets are assumed to be available at the sender prior to any transmissions. In a fixed generation-based linear network coding scheme, the sender chooses an integer $K \geq 1$, and sequentially partition the $N$ packets into $\left\lceil\frac{N}{K}\right\rceil$ generations $\left\{G_{1}^{f}, \cdots, G_{\left\lceil\frac{N}{K}\right\rceil}^{f}\right\}$, where $G_{i}^{f}=\left\{P_{i K+1}^{f}, \cdots, P_{\min ((i+1) K, N)}^{f}\right\}$. At each time slot $t$, the sender reads the head of the line (HOL) generation $G_{h}^{f}=$ $\left\{P_{h_{1}}^{f}, \cdots, P_{h_{K}}^{f}\right\}$, where $h=1, \cdots,\left\lceil\frac{N}{K}\right\rceil$ is the generation index, and $h_{k}, k=1, \cdots, K$ are the indices of packets within the generation. It then generates a coded packet $P[t]$ that is a linear combination of all packets in $G_{h}^{f}$ (shown in Figure 2), i.e.

$$
P[t]=\sum_{k=1}^{K} a_{k}[t] P_{h_{k}}^{f},
$$

where $a[t]=\left(a_{1}[t], \cdots, a_{K}[t]\right)$ is the coding coefficient vector, which is uniformly chosen at random from $\mathbb{F}_{q}^{K}[1]$. The coded packet, with the coefficient vector appended in the header, is then sent to the receiver through the erasure channel. The receiver collects coded packets over time. Given a large enough field $\mathbb{F}_{q}$, the receiver, with high probability [1], is able to decode the $K$ packets in the generation through Gaussian elimination performed on the linear system formed on any $K$ coded packets. Once the receiver decodes the HOL generation successfully, it sends an ACK message through the feedback channel to the sender. The sender, who receives the ACK after a delay of $D$ time slots, will purge the old HOL generation and move on to the next generation in the line.

Our scheme modifies such generation-based network coding in the following ways. The packets are not partitioned into generations prior to transmission. Instead, a coding bucket is created and acts like the HOL generation. We use the term bucket to avoid confusion with normal generation-based schemes. The size of the bucket in term of number of packets is denoted as $K$. The sender collects information about user-end delay constraints and chooses the bucket size $K$ dynamically. Figure 1(b) gives a simple example. At the beginning, the coding bucket contains three packets $\left\{P_{1}, P_{2}, P_{3}\right\}$. The sender keeps transmitting encoded packets, i.e. $P[1]$ to $P[5]$, of these three packets. Upon receiving the ACK feedback, it empties the bucket and decides to shrink bucket size to 2, possibly because of the tighter delay constraint experienced at the receiver. Therefore, only two packets $\left\{P_{4}, P_{5}\right\}$ go into the bucket for subsequent transmissions. We leave the details of adaptively determining coding bucket size to Section IV.

\section{B. $\ell_{p}$-Norm Delay Metrics}

Now we define the delay metrics used in the paper. Following the notations used in the previous part, let $T_{i}$ be the time slot in which the packet $P_{i}^{f}$ is decoded at the receiver, and is delivered to upper layer. We require the delivery of original data packet $\left\{P_{1}^{f}, \cdots, P_{N}^{f}\right\}$ to be in order. In the case when the sequence of packet decoded is out-of-order, 


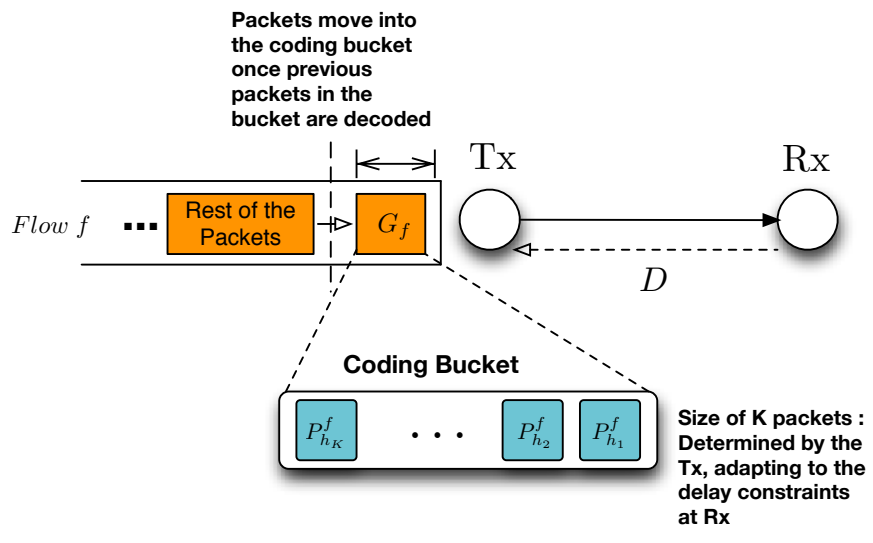

(a) Adaptive linear network coding based transmission model

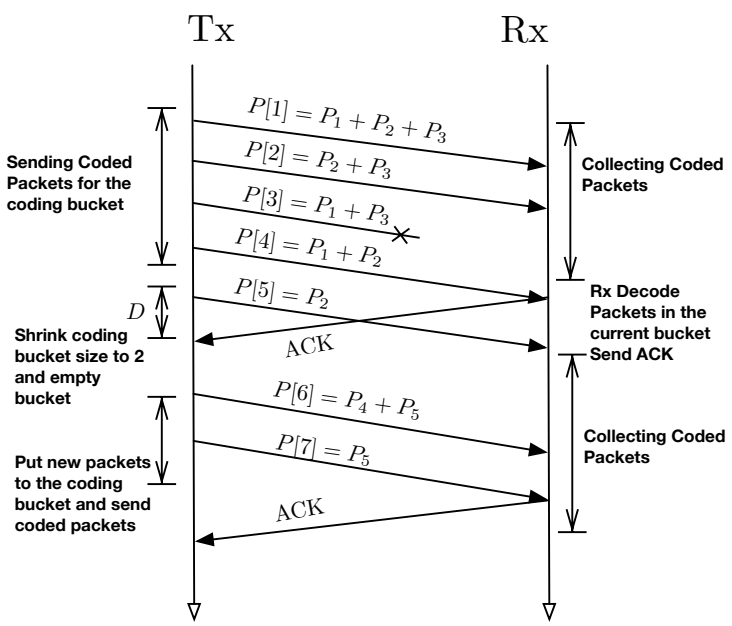

(b) A session with varying coding bucket size

Fig. 1. Adaptive Linear Coding Scheme in Point-to-Point Case

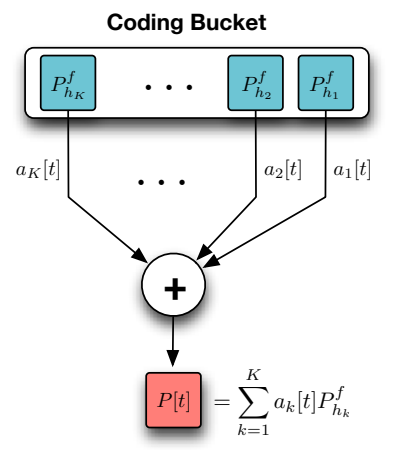

Fig. 2. An illustration of encoding

we assume that they are buffered at the receiver to ensure inorder final delivery. $T_{i}$ represents the final in-order delivery times of packets $P_{i}^{f}$, and we have $T_{1} \leq T_{2} \leq \cdots \leq T_{N}$. We define the inter-arrival times $\Delta T_{i}$ of the original packets to be:

$$
\begin{aligned}
& \Delta T_{1} \triangleq T_{1}+D \\
& \Delta T_{i} \triangleq T_{i}-T_{i-1}, \quad i=2, \cdots, N,
\end{aligned}
$$

where $D$ is the feedback delay from the receiver to the sender. Note that a feedback message $A C K$ is always assumed to be received correctly after $D$ time slots. However, when there is more than one receiver, we assume that, in general, receivers experience different feedback delays across the system owing to its location and channel variations. Let the size of each data packet be $L$. We define the delay cost function as a metric of the following form,

$$
d(p) \triangleq \frac{1}{L}\left(\frac{\sum_{i=1}^{N}\left(\mathbf{E}\left[\Delta T_{i}\right]\right)^{p}}{N}\right)^{1 / p}, \quad p \in[1, \infty),
$$

where $\mathbf{E}\left[\Delta T_{i}\right]$ is the expected value of $\Delta T_{i}$. The expectation is taken over the distribution of packet erasures over the system and all the randomness associated with the coding and scheduling scheme, which are specified in Section IV.

Mathematically, the delay metric is a normalized $\ell_{p}$-norm of the vector $\left[\mathbf{E}\left[\Delta T_{1}\right] \cdots \mathbf{E}\left[\Delta T_{N}\right]\right]^{T}$. Physically, however, $p$ mea- sures the delay sensitivity of the receiver and is predominantly dependent on the type of applications running on the receiver. As the value of $p$ varies from 1 to $\infty$, the delay function becomes increasingly biased towards the large components in the vector, hence indicating increasing user sensitivity toward large inter-packet delay. As an example, consider the case when $p=1$. Since $\sum_{i=1}^{N} \mathbf{E}\left[\Delta T_{i}\right]=\mathbf{E}\left[T_{N}\right]+D$, the delay in (4) simplifies to,

$$
d(1)=\frac{\mathbf{E}\left[T_{N}\right]+D}{L N},
$$

that is, $d(1)$ is the average delay per packet, normalized by the total size of the received data. Minimizing $d(1)$, therefore, is equivalent to average rate maximization for the receiver. On the other hand, consider the case when $p=\infty$. Because of the max norm, the delay function in (4) reduces to,

$$
d(\infty)=\frac{\max _{i} \mathbf{E}\left[\Delta T_{i}\right]}{L} .
$$

Effectively, minimizing $d(\infty)$ translates into minimizing the maximum expected inter-arrival time between any two successive packets. We call this the per-packet delay.

The flexibility in choosing various $p$-value for delay metrics provides a unified way of looking at the delay sensitivity at the user side. If a user is downloading a file, he is certainly more concerned about shortening the overall completion time or average delay per packet. Consequently, $d(1)$ is the appropriate delay metric to be optimized. On the other hand, if the user is running a real-time video applications, then $d(\infty)$ is more likely to be the right metric to be minimized as it allows sequence of packets to catch up quickly with respective delivery deadlines.

\section{Delay In Adaptive Coding Scheme}

In the adaptive coding scheme, a receiver will decode all packets in the current bucket before informing the sender to empty the bucket and move in new packets. Assume that the rate at which the coded packets are transmitted is $r$. Consider 
the transmission of a bucket of $K$ packets $\left\{P_{i_{1}}, \cdots, P_{i_{K}}\right\}$. Once the receiver collects $K$ linearly independent coded packets of the bucket, it decodes all $K$ packets together. Hence, the ordered inter-arrival times of original packets will satisfy, $\mathbf{E}\left[\Delta T_{i_{1}}\right]=\frac{K}{r}+D$ and $\Delta T_{i_{1}}=\cdots \Delta T_{i_{K}}=0$. In general, consider the case when the bucket size remains the same for a sequence of $N$ packets, $\left\{P_{i_{1}}, \cdots, P_{i_{N}}\right\} . N$ is divisible by $K$, as the bucket size may only change when the bucket is emptied. The packets will sequentially enter the bucket in groups of $K$ packets. Then, for the inter-arrival time of the $j$-th packet, we have,

$$
\mathbf{E}\left[\Delta T_{i_{j}}\right]=\left\{\begin{array}{ll}
\frac{K}{r}+D, & \text { if } j \equiv 1 \\
0, & \text { otherwise }
\end{array}(\bmod K),\right.
$$

Therefore, if the adaptive scheme chooses bucket size of $K$ of a sequence of $N$ packets, we can simplify (4) to measure the delay cost function for the transmission of the $N$ packets, resulting in:

$$
\begin{aligned}
d(p) & =\frac{1}{L}\left(\frac{\frac{N}{K} \sum_{j=1}^{K}\left(\mathbf{E}\left[\Delta T_{i_{j}}\right]\right)^{p}}{N}\right)^{1 / p} \\
& =\frac{1}{L}\left(\frac{\frac{N}{K}\left(\frac{K}{r}+D\right)^{p}}{N}\right)^{1 / p} \\
& =\frac{\frac{K}{r}+D}{L K^{1 / p}}
\end{aligned}
$$

In particular, under this coding scheme, the delay $d(p)$ seen by the receiver over the period is independent of $N$ as long as the coding bucket size remains to be $K$. Hence, we drop $N$ and only consider the bucket size $K$ for rest of the paper. Furthermore, in practice, $K$ takes only positive integer values in $\left[1, K_{\max }\right]$, where $K_{\max }$ is the maximum bucket size, limited by the maximum tolerable computation complexity of the target system. In this work, for simplicity, we assume that $K$ takes on real value in the same region $\left[1, K_{\max }\right]$.

\section{GEOMETRIC PROGRAMMING}

We give a concise primer of Geometric Programming before looking specifically into our system model. For more comprehensive coverage of the topic, we refer the reader to [17], [18]. Geometric program (GP) is a class of mathematical optimization problems characterized by some special forms of objective functions and constraints. A typical GP is nonlinear and non-convex, but can be converted into a convex program so that a local optimum is also a global optimum. The theory of GP has been well studied since the 60s [19]. Well developed solution techniques, such as interior point methods are capable of solving GPs efficiently even for large scale problems. Many high-quality GP solvers are available (e.g. MOSEK and CVX [20]) for providing robust numerical solutions for generalized GPs (GGP).

Consider a vector of decision variables $\mathbf{x}=\left[x_{1} \ldots x_{n}\right]^{T}$. A real function $g: \mathbf{R}^{n} \rightarrow \mathbf{R}$ is said to be a monomial if it can be written in the form,

$$
g(\mathbf{x})=c \prod_{i=1}^{n} x_{i}^{a_{i}}
$$

where the coefficient $c$ is positive, and the exponents $a_{1}, \ldots, a_{n}$ are arbitrary real numbers. A function $f: \mathbf{R}^{n} \rightarrow \mathbf{R}$ in the form

$$
f(\mathbf{x})=\sum_{k=1}^{K} c_{k} \prod_{i=1}^{n} x_{i}^{a_{i k}}
$$

with all $c_{k}$ being positive real numbers, is called a posynomial. A posynomial is the sum of arbitrary number of monomials. On top of this, any function $\tilde{f}$, which can be constructed with posynomials using addition, multiplication, positive power and maximum operations is called a generalized posynomial.

A standard form geometric program is presented as follows,

$$
\begin{array}{ll}
\operatorname{minimize} & f_{0}(\mathbf{x}) \\
\text { subject to } & f_{i}(\mathbf{x}) \leq 1, i=1, \ldots, m, \\
& g_{j}(\mathbf{x})=1, j=1, \ldots, p,
\end{array}
$$

where $f_{i}(x)$ are posynomials and $g_{i}(x)$ are monomials, and $x_{i}$ are the decision variables, which are also implicitly assumed to be positive, i.e. $x_{i}>0, i=1, \ldots, n$. In particular, the objective of the optimization has to be minimizing some posynomial. That says, for solving maximization problems with GP, the objective function has to be in the form of some monomial $g(\mathbf{x})$, so that instead of maximizing $g(\mathbf{x})$, we can minimize $\frac{1}{g(\mathbf{x})}$, which is itself a monomial. In the case where any $f_{i}(\mathbf{x})$ is a generalized posynomial, the optimization program is said to be a generalized geometric program (GGP). All generalized geometric programs can be converted into standard geometric programs and solved efficiently.

Note that a GP in its standard form is non-convex, as in general posynomials are non-convex. In order to apply general convex optimization methods, a GP is usually transformed into its convex form. Let $y_{i}=\log x_{i}$ so that $x_{i}=e^{y_{i}}$, the standard form GP can be transformed into its equivalent convex form,

$$
\begin{array}{ll}
\operatorname{minimize} & \log f_{0}\left(e^{\mathbf{y}}\right) \\
\text { subject to } & \log f_{i}\left(e^{\mathbf{y}}\right) \leq 1, i=1, \ldots, m, \\
& \log g_{j}\left(e^{\mathbf{y}}\right)=1, j=1, \ldots, p .
\end{array}
$$

In particular, a monomial constraints

$$
g_{j}(\mathbf{x})=d_{j} \prod_{k=1}^{n} x_{k}^{a_{j k}}=1
$$

is converted to

$$
\log g_{j}\left(e^{\mathbf{y}}\right)=\log d_{j}+\sum_{k=1}^{n} a_{i k} y_{k}=0,
$$

which is affine and convex. On the other hand, the posynomial parts are converted into log-sum-exp functions, which can be easily shown to be convex. Therefore, although the original standard formulations of GPs are nonlinear and non-convex, they can be converted into convex form as in (14) and solved 
efficiently. In this paper, we use GP to optimize the coding parameters and resource allocation at the transmitter with respect to the $\ell_{p}$-norm delay metric defined previously.

\section{Single Broadcast Channel With Packet ERASURES}

\section{A. System Model}

The motivating scenario of the work comes from a typical home network environment with multiple user networking devices. The receivers are wirelessly connected to a WiFi access point (AP), which is then linked with the gateway to the Internet. All the flow of packets from the Internet to the user devices goes through the gateway and the access point. The applications running on different devices have very different delay sensitivities and constraints, as discussed before. The gateway and the access point look for the optimal coding and scheduling parameters to ensure the $\mathrm{QoE}$ of all the users within the network.

Conceptually, we represent the system using the following model. We assume that the link between the AP and gateway has a high capacity and is lossless. Thus, we represent both the gateway and the AP together as a single node $s$. We denote the set of receivers by $T=\left\{t_{1}, \cdots, t_{M}\right\}$. Each receiver needs to obtain a flow of packets from some source over the Internet. Let $\mathcal{F}=\left\{f_{1}, \cdots, f_{M}\right\}$ be the set of flows, where $f_{i}$ is the packet flow requested by receiver $t_{i}$. Note that all $f_{i}$ enter the system from node $s$, which in turn acts as a source node. The flows for different receivers are assumed to be independent. The original data packets in each flow are numbered, with $P_{j}^{f_{i}}$ representing the $j$-th packet in flow $f_{i}$. We assume that there are always enough packets to be served for each flow, since that is the case when there is a heavy traffic condition. Furthermore, all packets are assumed to have the same size $L$ in the system and the system is time slotted. At any time slot, the node $s$ is able to broadcast a size $L$ packet to all receivers, through the packet erasure broadcast channel. Erasures happen independently across all receivers and all time slots, i.e. the channel is memoryless. We denote the erasure vector by $\mathbf{e}=$ $\left[\varepsilon_{1} \cdots \varepsilon_{M}\right]$, where $\varepsilon_{i}$ represents the erasure probability seen by receiver $t_{j}$. Figure 3 gives an illustration of the system model in the discussion.

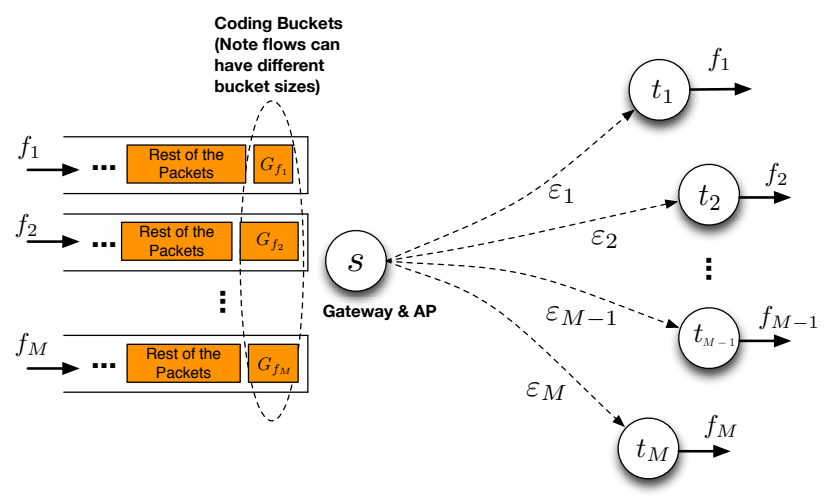

Fig. 3. System Model with Single Transmitter
1) Scheduling Strategies: Most of the works we discussed in Section $\square$ focus on linear network codes for multicast, in which all the receivers request the same content from the sources. In the system we consider here, however, we have a multiple unicast scenario, as each sink looks to receive its own flow, independently from others. The resource at node $s$ has to be shared among all the receivers. Specifically, for every time slot, the sender $s$ has to make a decision on which receiver to transmit to. While many sophisticated scheduling algorithms are available, for simplicity, we use a simple stochastic scheduling algorithm. At any time slot, the node $s$ serves receiver $t_{j}$ or flow $f_{j}$ with probability $a_{j}$, independently from of any other time slots. In the long run, equivalently, the transmitter node $s$ is spending $a_{j}$ portion of time serving receiver $t_{j}$. We call the vector $\mathbf{a}=\left(a_{1}, \cdots, a_{M}\right)$ the vector of scheduling coefficients.

2) Intra-session Coding: The adaptive coding scheme described in Section $\amalg$ is used in the system. In the multiple receiver case, we use intra-flow coding, i.e. each unicast flow is coded independently and separately from others. The coding bucket sizes and scheduling coefficients, however, are determined by solving system-wise optimizations. In this case, for a given time slot, if the transmitter decides to serve receiver $t_{j}$, it looks for packets in the coding bucket of flow $f_{j}$, and encodes these packets using random linear network codes. The coded packet is broadcasted to all the receivers. With probability $1-\varepsilon_{j}$, the targeted receiver $t_{j}$ will receive it correctly. Note that we assume the coding coefficients are embedded in the header of the packet and the size is negligible compared to the size of the packet $L$. The coding bucket size for flow $f_{j}$ is denoted as $K_{j}$. In general, $K_{i} \neq K_{j}$ for $i \neq j$, and $K_{j}$ may vary over time as the delay requirements at the receivers changes. Let $\mathbf{K}=\left(K_{1}, \cdots, K_{M}\right)$. We aim to optimize both $\mathbf{a}$ and $\mathbf{K}$, based on the varying delay constraints at the receivers.

\section{B. Delay Optimization}

We first consider the case where there is only a single receiver, i.e. $M=1$. Since there is no scheduling issue or system-wise fairness consideration in the case, it makes sense to minimize the delay cost function associated with the receiver. As there is no ambiguity of notations, we drop all the subscripts. It is easy to see that the packet transmission rate in this case is $1-\varepsilon$ for the receiver and thus the expected time for receiving $K$ coded packets is $\frac{K}{1-\varepsilon}$. Subsequently, the $\ell_{p}$-norm delay cost function minimization problem is given as follows,

$$
\begin{array}{cl}
\operatorname{minimize} & d(p)=\frac{\frac{K}{1-\varepsilon}+D}{L K^{1 / p}} \\
\text { subject to } & 1 \leq K \leq K_{\max } .
\end{array}
$$

The optimal block size $K^{*}$ can be obtained by setting zero to the gradient of the Lagrangian of objective function. We have

$$
K^{*}=\left(\frac{(1-\varepsilon) D}{p-1}\right)_{\left[1, K_{\max }\right]}, \quad 0<\varepsilon<1,
$$


where the subscript denotes the projection,

$$
(x)_{[a, b]} \triangleq \min (\max (a, x), b) .
$$

For better understanding of the delay metrics, consider the relation between $d(1)$ and $d(\infty)$. From (4), we have,

$$
K=\frac{D(1-\varepsilon)}{(1-\varepsilon) L d(1)-1} .
$$

Hence, the trade-off between $d(1)$ and $d(\infty)$ can be expressed as follows,

$$
d(\infty)=\frac{D}{L-\frac{1}{d(1)(1-\varepsilon)}} .
$$

Ignoring the bucket size constraints for simplicity, given $D$, we can vary $K$ from 1 to $\infty$, and plot the values of $d(\infty)$ against $d(1)$ for the trade-off curve. Each point on the curve corresponds to a choice of $K$, which is equivalent to a choice of optimizing $d(p)$ for some $p$, because of (18). Therefore, the choice of $p$ at the receiver indicates the a point on the trade-off curve of $d(1)$ and $d(\infty)$ that is desired by the receiver.

We can also use the zero duality gap in GP to obtain the optimal $d(p)$ directly from the dual function. Note that the dual function of (16) is given by,

$$
v=\left(\frac{1}{(1-\varepsilon) L \beta_{1}}\right)^{\beta_{1}}\left(\frac{D}{L \beta_{2}}\right)^{\beta_{2}}
$$

where $\beta=\left(\beta_{1}, \beta_{2}\right)$, can be obtained from solving a simple linear system,

$$
\left\{\begin{array}{lll}
(1-1 / p) \beta_{1}+(-1 / p) \beta_{2} & =0, & \text { (normality condition) } \\
\beta_{1}+\beta_{2} & =1, & \text { (orthogonality condition) }
\end{array}\right.
$$

\section{Delay Constrained Optimization with GP}

1) GP Formulation: For $M>1$, instead of minimizing the delay of a specific receiver, we are interested in optimizing certain system-wise utility function with the constraints that the $\ell_{p}$-norm delay requirements must be satisfied at each receiver. We assume the each receiver $t_{j}$ monitors the delay constraints for targeted QoE of its applications and set a maximum acceptable delay $\hat{d}\left(p_{j}\right)$, corresponding to its delay sensitivity $p_{j}$. For the objective function, we choose to maximize the min rate of all receivers. If the packet transmission rate to $t_{j}$ is $r_{j}$, then the actual data rate received by $t_{j}$ is $\frac{L K_{j}}{\frac{K_{j}}{r_{j}}+D_{j}}$, where $D_{j}$ is the feedback delay of $t_{j}$. Let $\mathbf{r}=\left(r_{1}, \cdots, r_{M}\right)$. The optimization problem is then given as follows:

$$
\begin{array}{lll}
\max _{\mathbf{K}, \mathbf{r}, \mathbf{a}} & \min _{j} \frac{L K_{j}}{\frac{K_{j}}{r_{j}}+D_{j}} & \\
& & \frac{K_{j}}{r_{j}}+D_{j} \\
\text { subject to } & \frac{L \hat{d}_{j}\left(p_{j}\right)}{L K_{j}^{1 / p_{j}}} & \forall j=1, \ldots, M \\
& r_{j} \leq a_{j}\left(1-\varepsilon_{j}\right) & \forall j=1, \ldots, M \\
& \sum_{j} a_{j} \leq 1 & \\
& 1 \leq K_{j} \leq K_{\max } & \forall j=1, \ldots, M .
\end{array}
$$

In the above formulation, constraints (24) and (25) represent the delay and rate constraints respectively for receiver $t_{j}$, while (26) is the scheduling probability constraint at the sender node $s$. The problem is a Generalized Geometric Program. In particular, all constraints can be converted into upper bound of posynomials of $\mathbf{K}, \mathbf{r}$ and $\mathbf{a}$. The only non-posynomial part is the objective function, which can be transformed into upper bounding posynomial constraints and monomial objective by adding auxiliary variable $x$,

$$
\begin{array}{rl}
\max _{\mathbf{K}, \mathbf{r}, \mathbf{a}, x} & x \\
\text { subject to } & \frac{x\left(\frac{K_{j}}{r_{j}}+D_{j}\right)}{L K_{j}} \leq 1, \quad \forall j .
\end{array}
$$

Combining this with (24) to (27), we have a GP that can be efficiently solved.

\section{Illustrations of Trade-offs}

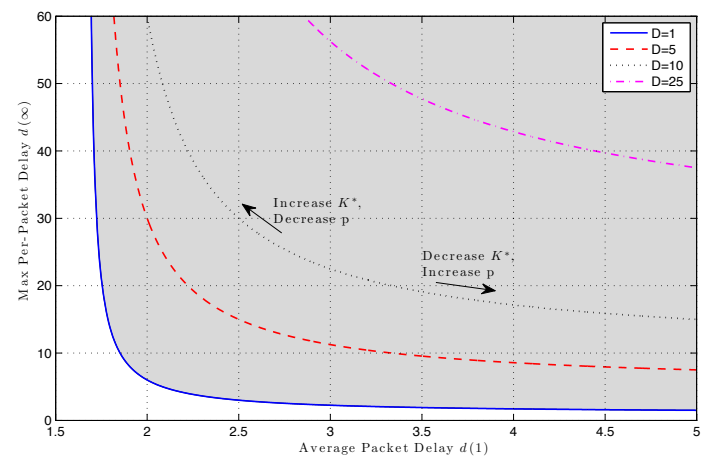

Fig. 4. Tradeoff of $d(1)$ vs $d(\infty)$ with varying $D$

1) Trade-off: Average Delay vs Per-packet Delay: Figure 4 demonstrate the trade-off between $d(1)$ and $d(\infty)$ following Equation (20) with various values of $D$ and erasure probability $\varepsilon=0.4$. As discussed previously, if we parameterize $d(1)$ and $d(\infty)$ on the optimal bucket size $K^{*}$, as $p$ varies from 1 to $\infty$, we obtain the same curves. The shaded area bounded by each curve is the area of all achievable pairs $(d(1), d(\infty))$ for the specific feedback delay. With small $D$, both low delay in $d(1)$ and $d(\infty)$ can be achieved. However, when feedback delay increases, the trade-off becomes increasingly stronger. This is evident from Equation (20), where $D$ appears in the numerator. It is expected as for average delay, coding over larger generations amortizes the feedback delay over more packets. But for the per-packet delay $d(\infty)$, increased feedback delay must be compensated by even smaller generation size for more frequent decoding. This is also consistent with Equation (18) where $K^{*}$ increases with feedback delay $D$ and decreases as delay sensitivity $p$.

2) Adaptive Scheme vs Fixed Generation Coding: Figure 5 to 7 shows some comparisons between adaptive coding schemes with fixed generation size coding schemes, as the delay sensitivity $p_{1}$ of the first receiver increases. In this example, we have 5 receivers, with erasure $\mathbf{e}=$ $[0.4,0.1,0.15,0.2,0.25]$, the same $D=5, L=1$ and 


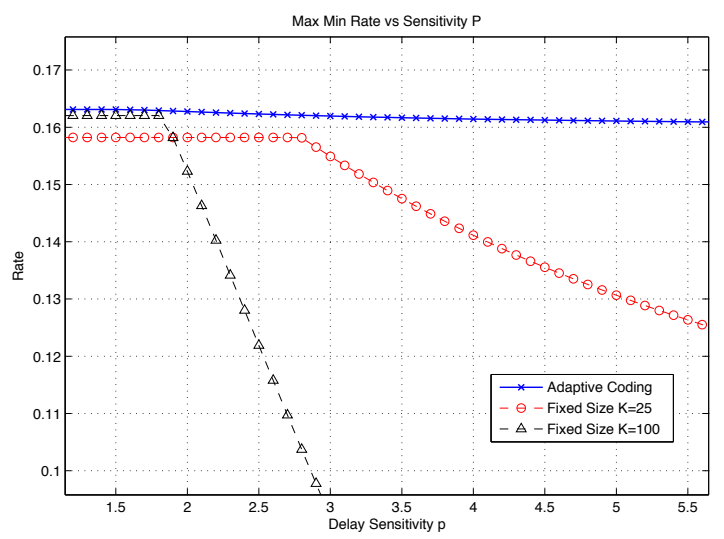

Fig. 5. Min Rate vs Delay Sensitivity $p_{1}$

$\hat{d}_{j}=50 / L$. Except for receiver 1 , whose $p_{1}$ value varies, we have $p_{j}=1$ for all other receivers. For the fixed generation size schemes, we choose $K=25$ and $K=100$ for representing small and large generation respectively. In this cases, scheduling coefficients are the only decision variables in the optimization for min rate. From Figures 5 and 6 we can see that, initially, the min rates for different schemes are relatively close. The adaptive scheme is able to choose a much larger coding bucket size to obtain some rate gain compared to $K=25$ case. As $p_{1}$ increases, the fixed coding generation schemes are unable to reduce generation size. In order to meet the growingly stringent delay constraint, the sender has to devote increasingly more time to receiver 1 , as seen in Figure 7 Inevitably, the time for serving other receivers is greatly reduced and the min rate of the system decreases quickly. In the $K=100$ case, the delay requirements cannot be satisfied for $p_{1}>2.9$. On the contrary, for the adaptive scheme, which optimizes bucket size and scheduling jointly, there is little decrease in min rate. For low delay sensitive receivers, the scheme will assign them large coding bucket sizes to allow rate gain. As a results, the sender is able to meet their delay-rate constraints with less serving time and save time for higher receivers. On the other hand, as $p$ values for some receiver increases, its coding bucket size is reduced to quickly decreases the per-packet delay. Hence, the scheme is able to accommodate high delay sensitive receivers much better.

\section{Multiple Wireless Packet Erasure Channels}

With the proliferation of low cost access points, many devices may be covered by more than one access points in wireless home, campus or enterprise networks. That leads to an important extension of the work to the case of multiple broadcast erasure channels covering the same set of receivers. As in the previous section, we still have the same set of receivers, $T=\left\{t_{1}, \cdots, t_{M}\right\}$. However, there are now $W$ access points, or transmitters, denoted by the set $S=\left\{s_{1}, \cdots, s_{W}\right\}$. Instead of an erasure probability vector, we have an erasure probability matrix $\mathbf{e}=\left[\varepsilon_{i j}\right]$, where $\varepsilon_{i j}$ is the erasure probability between node $s_{i}$ and $t_{j}$. An example of the system is illustrated in

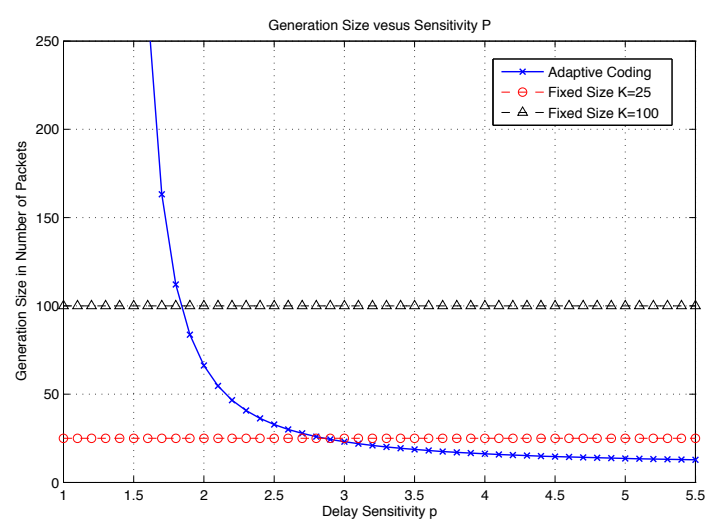

Fig. 6. Coding Bucket Size vs Delay Sensitivity $p_{1}$

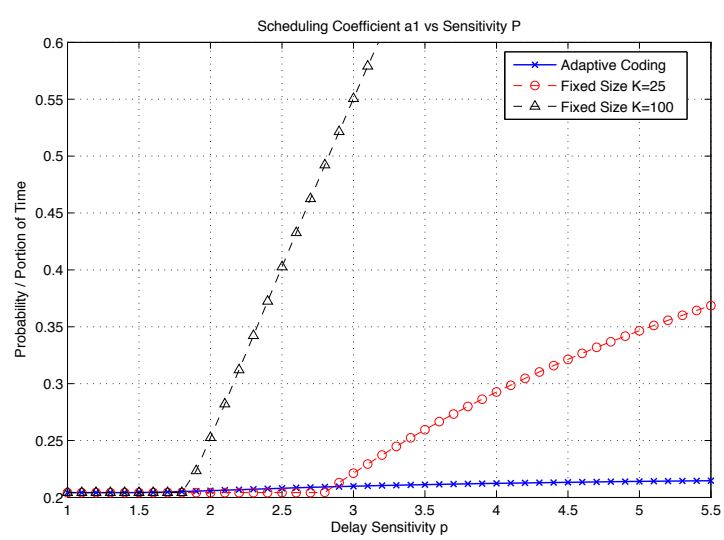

Fig. 7. $a_{1}$ vs Delay Sensitivity $p_{1}$

Figure 8 . We assume that the channels are orthogonal or noninterfering.

The same coding and scheduling scheme is used for the new system. We use $\mathbf{a}=\left[a_{i j}\right]$ to represent the probability of transmitter $s_{i}$ serving the flow $f_{j}$ at any time slot. The scheduling and coding optimization is done at the gateway

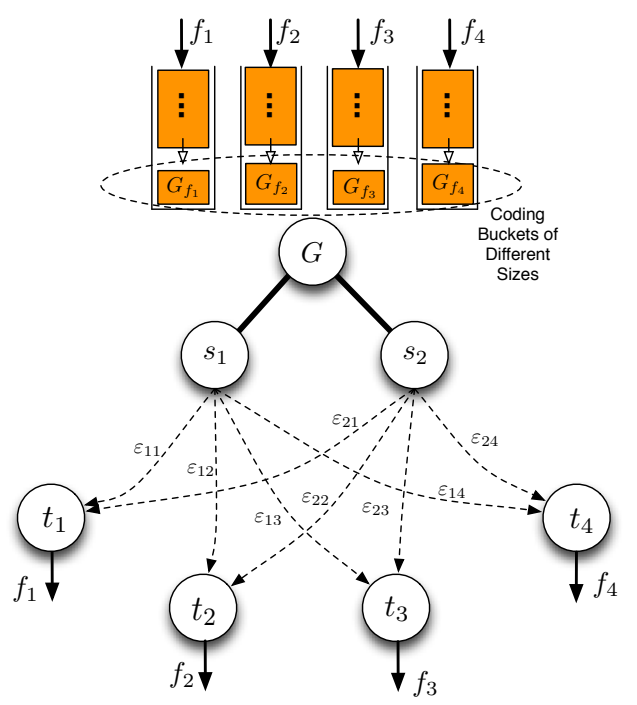

Fig. 8. An example system with 2 senders and 4 receivers 
node $G$, who coordinates all senders who perform encoding. Furthermore, for each flow $f_{j}$ in $\mathcal{F}$, all senders in $S$ have the same coding bucket size $K_{j}$, dictated by node $G$. This ensures that, for each flow, every sender sends coded packets in the coding buckets consisting of the same data packets and guarantees the decodability. An important feature of the randomly linear coding is that all coded packets from the same bucket are exchangeable. That avoids complicated scheduling based on sequence numbers of the uncoded packets and helps to reduce transmission redundancy in erasure channels.

\section{A. Signomial Program Formulation}

Similarly to the single sender case, we can formulate an optimization program for determining $\mathbf{K}, \mathbf{a}$ and $\mathbf{r}$. For example, the rate product maximization is given as,

$$
\begin{aligned}
& \min _{\mathbf{K}, \mathbf{r}, \mathbf{a}, \mathbf{R}} \prod_{j} R_{j}^{-1} \\
& \text { subject to } \frac{\frac{K_{j}}{r_{j}}+D_{j}}{L K_{j}^{1 / p_{j}}} \leq \hat{d}_{j} \quad \forall j=1, \ldots, M \\
& r_{j} \leq \sum_{i} a_{i j}\left(1-\varepsilon_{i j}\right) \quad \forall j=1, \ldots, M \\
& R_{j} \leq \frac{L K_{j}}{\frac{K_{j}}{r_{j}}+D_{j}} \quad \forall j=1, \ldots, M \\
& 1 \leq K_{j} \leq K_{\max } \quad \forall j=1, \ldots, M \\
& \sum_{j} a_{i j} \leq 1 \quad \forall i=1, \ldots, W,
\end{aligned}
$$

where the delay constraint (31) and complexity constraint 34 remain the same. Auxiliary variables $R_{j}$ and (33) are used to represent the average rates for the receiver. Maximizing rate product is equivalent to minimizing the product of average delays $R_{j}^{-1}$, hence the objective $\prod_{j} R_{j}^{-1}$. The packet transmission rate for each receiver in this case is bounded by $\sum_{i} a_{i j}\left(1-\varepsilon_{i j}\right)$. However, owing to the existence of this new transmission rate constraint (32), the problem becomes truly non-convex. In particular, the constraint can be written as

$$
r_{j}+\sum_{i}\left(-a_{i j}\right)\left(1-\varepsilon_{i j}\right) \leq 0,
$$

which is an upper bound constraint on a signomial. A signomial is a sum of monomials whose multiplicative coefficients can be either positive or negative. The problem therefore belongs to a more general class of problem called Signomial Program, which is truly non-convex and NP-hard in general. Only local optimal solutions can be efficiently computed. Based on the most widely used monomial condensation methods, we provide an efficient way to approximate the solution with successive GP solutions.

\section{B. Successive GP Approximation}

Consider an arbitrary signomial $h(\mathbf{x})$. It can always be written as the difference between two posynomials, i.e. $h(\mathbf{x})=$ $f^{+}(\mathbf{x})-f^{-}(\mathbf{x})$. The inequality $h(\mathbf{x}) \leq 0$ is then equivalent to $\frac{f^{+}(\mathbf{x})}{f^{-}(\mathbf{x})} \leq 1$. We can approximate the left hand side with a posynomial using common condensation methods [18]. In single condensation, the posynomial denominator $f^{-}(\mathbf{x})$ is approximated using a monomial $g^{-}(\mathbf{x})$, which in turn allows $\frac{f^{+}(\mathbf{x})}{f^{-}(\mathbf{x})}$ to be approximated by a posynomial $\frac{f^{+}(\mathbf{x})}{g^{-}(\mathbf{x})}$. In double condensation, both $f^{+}$and $f^{-}$are approximated using monomials, which creates a monomial approximation of $\frac{f^{+}(\mathbf{x})}{f-(\mathbf{x})}$. In our case, both methods are equivalent, since we have $f^{+}(\mathbf{x})=r_{j}$, which is itself a monomial. One of the commonly used condensation methods is based on the following Lemma [18].

Lemma 1: Given a posynomial $f(\mathbf{x})=\sum_{i} u_{i}(\mathbf{x})$, choose $\beta_{i}>0$, such that $\sum_{i} \beta_{i}=1$, then the following bound holds,

$$
f(\mathbf{x}) \geq g(\mathbf{x})=\prod_{i}\left(\frac{u_{i}(\mathbf{x})}{\beta_{i}}\right)^{\beta_{i}} .
$$

Furthermore, equality holds when $\mathbf{x}=\mathbf{x}_{0}$ and $\beta_{i}=\frac{u_{i}\left(\mathbf{x}_{0}\right)}{f\left(\mathbf{x}_{0}\right)}$.

Proof: The results can be easily proved using Inequality of Arithmetic and Geometric Mean (AM-GM).

Using Lemma 1 we can approximate constraint (32) in the signomial program with the following,

$$
r_{j} \leq \prod_{i}\left(\frac{a_{i j}\left(1-\varepsilon_{i j}\right)}{\beta_{i j}}\right)^{\beta_{i j}} .
$$

In particular, the optimization program is then a Geometric Program, if we replace (32) with (38). Furthermore, given the monomial approximation in (38), we can construct successive GP based on refined approximations of constraint (32) to approach local optimal solutions of the original Signomial Problem. The algorithm is summarized in Algorithm 1.

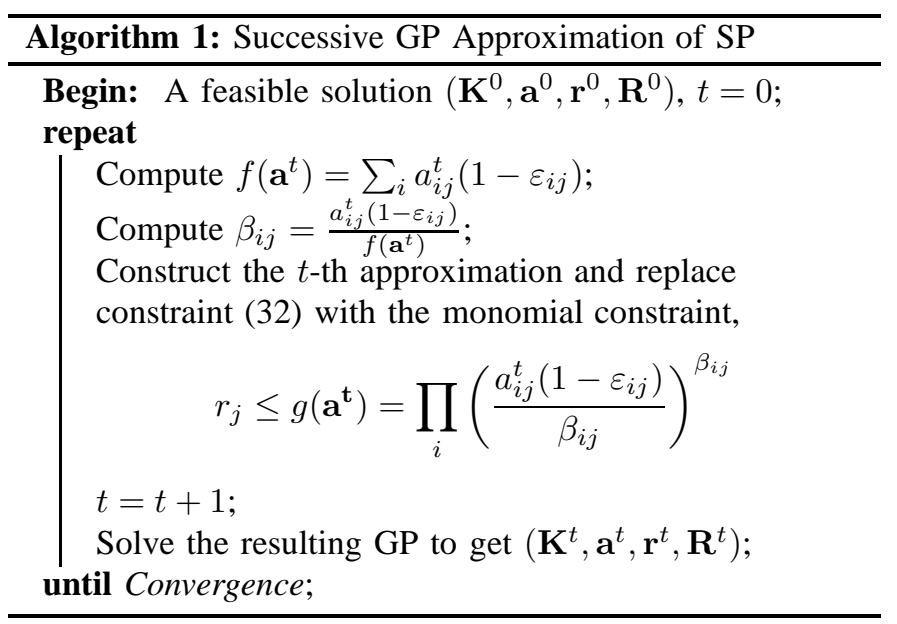

\section{Convergence}

Given Lemma 1 it is easy to show the Algorithm 1 always converges. According to Lemma 1, the values $\beta_{i j}$ are chosen such that for the local approximation at $\mathbf{a}^{t}$ in the $t$-th iteration, we have,

$$
g\left(\mathbf{a}^{t}\right)=f\left(\mathbf{a}^{t}\right) \geq f\left(\mathbf{a}^{t-1}\right) .
$$

Let the optimal objective for iteration $t$ be $Z^{*, t}$. Then we have $Z^{*, t} \leq Z^{*, t-1}$. Furthermore, at local optimal $\mathbf{a}^{*}$, it can 
be verified, that $f\left(\mathbf{a}^{*}\right)=g\left(\mathbf{a}^{*}\right)$ and $\nabla f\left(\mathbf{a}^{*}\right)=\nabla g\left(\mathbf{a}^{*}\right)$, which shows that the algorithm will indeed converge to an optimal that satisfies the KKT condition. In fact, in many cases, it converges to the global optimum. Figure 9 and 10 shows the convergence of min rate and bucket sizes for a example system with 3 receivers and 2 transmitters.

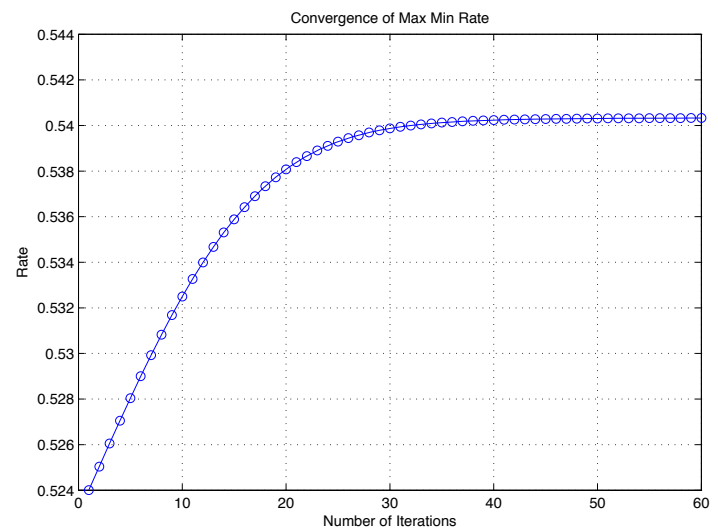

Fig. 9. Convergence of Optimal Min rate in the example system

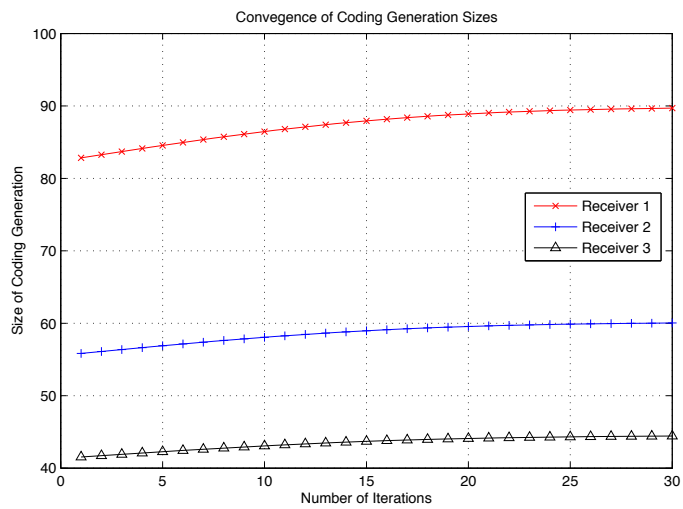

Fig. 10. Convergence of Bucket Sizes in the example system

\section{Conclusions}

In this paper, we consider the trade-off between rate and delay in single-hop packet erasure broadcast channels with random linear coding schemes. We characterize the delay and rate requirements of various users with a unified framework based on the $\ell_{p}$-norm delay metrics defined on the in-order packet arrival times. Using the optimal trade-off curve between the average delay, which can be viewed as the inverse of rate, and the per-packet delay, we demonstrate how feedback delays and the choice of coding bucket sizes affect the tradeoffs. In the multiple receiver case, we formulate geometric optimization problems to exploit the trade-off together with the transmission time allocate at the senders. With an adaptive coding scheme, for low delay sensitive receiver, the sender could allocation less time while compensating the rate loss with larger coding bucket. That allows the sender to allocate more time to high delay sensitive receivers who, at the same time, are assigned with smaller coding bucket sizes. We show that the adaptive scheme is more robust and resilient toward high and varying delay sensitivities, since the feedback information about receiver delay constraints adds extra flexibility to the coding and scheduling design. In particular, in many systems, this comes with little cost because of the availability of feedback channels. Finally, when there are multiple senders, we formulate the same optimization problem into a nonconvex signomial problem and approximate the solution with successive GP approximations based on single condensation methods and we demonstrate the convergence of the algorithm.

\section{REFERENCES}

[1] T. Ho, M. Médard, R. Koetter, D. R. Karger, M. Effros, J. Shi, and B. Leong, "A random linear network coding approach to multicast," IEEE Transactions on Information Theory, vol. 52, no. 10, pp. 44134430, Oct. 2006.

[2] P. Chou, Y. Wu, and K. Jain, "Practical network coding," in Proceedings of the Annual Allerton Conference on Communication Control and Computing, vol. 41, 2003, pp. 40-49.

[3] J. MacLaren Walsh, S. Weber, and C. wa Maina, "Optimal Rate-Delay tradeoffs and delay mitigating codes for multipath routed and network coded networks," IEEE Transactions on Information Theory, vol. 55, no. 12, pp. 5491-5510, Dec. 2009.

[4] Y. Li, M. Chiang, A. R. Calderbank, and S. N. Diggavi, "Optimal Rate-Reliability-Delay tradeoff in networks with composite links," IEEE Transactions on Communications, vol. 57, no. 5, pp. 1390-1401, May 2009.

[5] R. Ahlswede, N. Cai, S. Li, and R. Yeung, "Network information flow," Information Theory, IEEE Transactions on, vol. 46, no. 4, pp. 12041216, 2000.

[6] S. Y. Li, R. W. Yeung, and N. Cai, "Linear network coding," IEEE Transactions on Information Theory, vol. 49, no. 2, pp. 371-381, Feb. 2003.

[7] R. Koetter and M. Médard, "An algebraic approach to network coding," Networking, IEEE/ACM Transactions on, vol. 11, no. 5, pp. 782-795, 2003.

[8] M. Luby, "LT codes," in The 43rd Annual IEEE Symposium on Foundations of Computer Science, 2002. Proceedings. IEEE, 2002, pp. 271280.

[9] A. Shokrollahi, "Raptor codes," IEEE Transactions on Information Theory, vol. 52, no. 6, pp. 2551-2567, Jun. 2006.

[10] D. J. MacKay, "Fountain codes," Communications, IEE Proceedings-, vol. 152 , no. 6 , pp. 1062- 1068, Dec. 2005.

[11] A. Eryilmaz, A. Ozdaglar, M. Médard, and E. Ahmed, "On the delay and throughput gains of coding in unreliable networks," Information Theory, IEEE Transactions on, vol. 54, no. 12, pp. 5511-5524, 2008.

[12] D. Nguyen, T. Tran, T. Nguyen, and B. Bose, "Wireless broadcast using network coding," IEEE Transactions on Vehicular Technology, vol. 58, no. 2, pp. 914-925, Feb. 2009.

[13] J. K. Sundararajan, P. Sadeghi, and M. Médard, "A feedback-based adaptive broadcast coding scheme for reducing in-order delivery delay," in Workshop on Network Coding, Theory, and Applications, 2009. NetCod '09. IEEE, Jun. 2009, pp. 1-6.

[14] L. Keller, E. Drinea, and C. Fragouli, "Online broadcasting with network coding," in Fourth Workshop on Network Coding, Theory and Applications, 2008. NetCod 2008. IEEE, Jan. 2008, pp. 1-6.

[15] P. Sadeghi, D. Traskov, and R. Koetter, "Adaptive network coding for broadcast channels," in Workshop on Network Coding, Theory, and Applications, 2009. NetCod '09. IEEE, Jun. 2009, pp. 80-85.

[16] D. S. Lun, P. Pakzad, C. Fragouli, M. Médard, and R. Koetter, "An analysis of Finite-Memory random linear coding on packet streams," in 2006 4th International Symposium on Modeling and Optimization in Mobile, Ad Hoc and Wireless Networks. IEEE, Apr. 2006, pp. 1- 6.

[17] S. Boyd, S. Kim, L. Vandenberghe, and A. Hassibi, "A tutorial on geometric programming," Optimization and Engineering, vol. 8, pp. 67127, Apr. 2007.

[18] M. Chiang, Geometric programming for communication systems. Now Publishers Inc, 2005.

[19] R. Duffin, E. Peterson, and C. Zener, Geometric programming: theory and application. Wiley, 1967.

[20] M. Grant and S. Boyd, "CVX: matlab software for disciplined convex programming," 2008. 